\title{
Research on Crease Detection of Feather's Quill Based on Coupling Shock Filter and Complex Diffusion
}

\author{
He zuihong \\ School of Automation \\ Guangdong University of Technology \\ Guangzhou,China \\ Experiment Center \\ Guangzhou University \\ Guangzhou,China \\ e-mail:2243322408@qq.com
}

\author{
Wang Renhuang \\ School of Automation \\ Guangdong University of Technology \\ Guangzhou,China \\ e-mail: wrh@gdut.edu.cn
}

\begin{abstract}
In crease detection of feather's quill, aim at the phenomenon that noise is also enlarged during the course of enhancing crease edge, a method of crease detection for feather's quill based on coupling shock filter and complex diffusion is proposed. The method based on the fact that shock filter can sharpen and enhance image edge and the real part of complex diffusion can reduce the noise, meanwhile the imaginary part can detect edge. Then the idea of coupling shock filter and complex diffusion is adopted. In the coupling process, shock flow velocity is controlled by the second derivative of image and time dependency is incorporated to the process. This lead to the result that shock filter is controlled by the imaginary part of complex diffusion. By comparing the simulation results, it is seen that with this method the real part and the imaginary part of complex diffusion can both effectively reduce the noise, and the imaginary part can also better enhance image edge. The experimental results show that the method improved significantly the visual quality of feather's quill image and can effectively detect the crease defects.
\end{abstract}

Keywords-shock filter; complex diffusion; image denoising; image enhancement; edge detection

\section{INTRODUCTION}

Feather used for badminton is all processed from natural duck feather or goose feather. Due to the nature of feather, there are dozens of pinaculum defects that are quite common, such as deficiency, shortage of filament, moth damage, ripple, wrinkled feather, scar, crease, blot, heterochrome of feather and integral color deviation. Badminton production in China has occupied over $90 \%$ of the total in the world.Currently the sorting and choosing of feather mainly by manual work costs a great deal of labor force, which directly affect quality of the badminton and the benefit of the manufacturer. Machine vision based feather detection will be the foundation for badminton production automation. This paper discusses sorting and choosing of feather quills' crease defect that is a key index for badminton's endurance in performance. Realizing automatic sorting and choosing offers a significant and practical value to improvement of badminton's quality.

There existing various of crease defect. It is hard to achieve sound effect by simple processing.After a lot of crease defect analysis and calculation we get crease approximately shown as a straight line with certain width and length, which is accordance with curve of feather quill. Crease in the image only has a very small gray difference with neighboring areas, but changed shapes of internal part of crease leads to brightness difference which that can be an edge to recognize crease. When brightness difference is small, the crease edge is too weak to detect, needing calculation to enhance crease edge. What's more, due to the impact of the imaging conditions and the limitation of imaging equipment, crease edge gained is blurred and image is contained with noise. Traditional method to get rid of noise through image enhancement based on point operation and neighboring operation cannot remove noise when enhance the edge. Because of the image noise and edge are all belong to the category of high frequency signal, therefore it is also difficult to realize image enhancement by filtering in the scope of frequency area.

Based on the crease defects and its edge features, as well as the analysis of shortcomings of shock filter model and Gilboa complex diffusion model, this thesis puts forward a method of coupling shock filter and complex diffusion to remove noise and extract edge for crease defect image. This method is used to synthetic crease image, along with comparing the simulation results to demonstrate the advantages and disadvantages of this method, and the model parameters are determined. At last the method is applied to crease detection of feather quill, validity is verified through experiments.

\section{MODEL OF SHOCK FILTER}

In 1990, Rudin and Osher in reference [1] proposed shock filter model for image enhancement. This model takes fuzzy signal as initial value of equation and solution of equation is a function related with time. When $t \rightarrow \infty$, solution of equation $I(\cdot, t)$ constringes, and value tends to be stable state, at the same time, solution of partial differential equation meets the maximum - the minimum principle. For 2D signal, model of shock filter is:

$$
\left\{\begin{array}{l}
I_{t}=-\operatorname{sign}\left(I_{\eta \eta}\right)|\nabla I| \\
I(x, y, 0)=I_{0}(x, y)
\end{array}\right.
$$


Where $\eta$ is the gradient direction of image, $I_{\eta \eta}$ is the second derivative of the gradient direction of image and $\operatorname{sign}\left(I_{\eta \eta}\right)$ is edge detection operator. Image sharpen and enhancement based on shock filter have the following features: (1) image enhancement takes place in original image's pixel of zero crossing point (inflection point), where $I_{\eta \eta}=0$. (2) partial extreme value of the enhanced image remains unchanged. (3) shock filter model's steady state solution (weak solution) is a piecewise constant function. (4) image enhancement process is similar to a process of deconvolution.

\section{MODEL OF COMPLEX DIFFUSION}

\section{A. Linear complex diffusion}

Linear complex diffusion is defined as:

$$
\begin{cases}I_{t}=c I_{x x}=c \Delta I, & t>0, \quad x \in \square \\ I(x, 0)=I_{0} \in \square, & c, I \in \square\end{cases}
$$

Where $c$ is diffusion coefficient, if $c \in R$ then formula (2) is linear solid diffusion equation. While $c>0$ this process is a steadily forward diffusion, while $c<0$ the process is a pathological reverse diffusion.

Suppose complex diffusion coefficient $c=r e^{i \theta}$ due to the inverse diffusion process does not exist stable basic solution, take the real part of $c$ as positive case, namely $\theta \in(-\pi / 2, \pi / 2)$. For any $t \geq 0$, when $\theta \rightarrow 0$, the approximate solution of formula (2) is ${ }^{[2,3]}$ :

$$
\begin{aligned}
& \lim _{\theta \rightarrow 0} \operatorname{Im}(I) / \theta=t \Delta g_{\sigma} * I_{0} \\
& \lim _{\theta \rightarrow 0} \operatorname{Re}(I)=g_{\sigma} * I_{0}
\end{aligned}
$$

Where, $\sigma=\lim _{\theta \rightarrow 0} \sigma=\sqrt{2 t}$.

Formula (3), (4) shows that when $\theta$ is small enough, the real part of solution to equation (2) is approximate to the Gaussian convolution of $I_{0}$, which can remove noise, while the imaginary part $\operatorname{Im}(I) / \theta$ is approximate to Laplace operator of $I_{0}$ Gaussian convolution (take time $t$ as scale). Therefore, the imaginary part which is a smooth second derivative can be used as edge detection operator, and it will changes its scale with time, which is similar to the gradient role in the real number field. The diffusion process can be controlled by the imaginary part (divided exactly by $\theta$ ) of the image.

\section{B. Nonlinear complex diffusion}

In 2003, Guy Gilboa put forward nonlinear complex diffusion, with its diffusion coefficient in plural form, and applied it to remove Gaussian white noise, the definition is as follows ${ }^{[2]}$ :

$$
\left\{\begin{array}{l}
I_{t}=\nabla \cdot(c(\operatorname{Im}(I)) \nabla I)=\operatorname{div}(c(\operatorname{Im}(I)) \nabla I) \\
c(\operatorname{Im}(I))=e^{i \theta} / 1+(\operatorname{Im}(I) / k \theta)^{2}
\end{array}, c, I \in \square\right.
$$

Where $k$ is the threshold parameter, $\operatorname{Im}(I) / \theta$ depicts details information of image's edge, which is similar to the gradient role in the real number field. $\theta$ Should fully be small ( $\theta \square 1^{\circ}$ ); and the real part of diffusion function $\operatorname{Re}(I)$ is smoothed image. But in the experiment, we found that this model is still very sensitive to noise. After nonlinear complex diffusion the real part of image which means the edge and stripe is polished, while the imaginary part shows noise amplification.

\section{MODEL OF COMPLEX SHOCK FILTER}

\section{A. Coupling shock filter and diffusion}

Alvarez and Mazorra added Gaussian filter and anisotropic diffusion part in the shock filter formula (1) and put weighting function on diffusion term, namely coupling shock filter and diffusion operator, finally the following ALV model [5] is put forwarded:

$$
I_{t}=-\operatorname{sign}\left(G_{\sigma} * I_{\eta \eta}\right)|\nabla I|+c I_{\xi \xi}
$$

Where, $\xi$ is the tangential direction perpendicular to $\nabla I, I_{\xi \xi}$ is second derivative in the tangential direction, $c$ is a positive constant functioning to balance the effect between diffusion term and shock term. This model introduces diffusion term of tangential direction to restrain noise and the smooth of image has always been there in the process of image processing, which lead to the loss of detail information.

Coulon and Arridge put forward the CA mode [6] as follows:

$$
I_{t}=\operatorname{div}(c \nabla I)-(1-c)^{\alpha} \operatorname{sign}\left(G_{\sigma} * I_{\eta \eta}\right)|\nabla I|
$$

Where, $\quad c=\exp \left(-\left|G_{\sigma} * \nabla I\right|^{2} / k\right), \quad k \quad$ is gradient threshold, $\alpha$ is positive number, the de-noising part of the first term is equation $\mathrm{P}-\mathrm{M}$ [4]. The second term is edge enhancement. Weight $(1-c)^{\alpha}$ prevents this process from vibrating in the point of noise and the bigger $\alpha$ is, the stronger the control has.

Kornprobst and others propose model as follows [7]:

$I_{t}=\alpha_{\gamma}\left(h_{\tau} I_{\eta \eta}+I_{\xi \xi}\right)-\alpha_{e}\left(1-h_{\tau}\right) \operatorname{sign}\left(G_{\sigma} * I_{\eta \eta}\right)|\nabla I|$

Where, $h_{\tau}=h_{\tau}\left(\left|G_{\sigma} * \nabla I\right|\right)= \begin{cases}1 & \text { if }\left|G_{\sigma} * \nabla I\right|<\tau \\ 0 & \text { otherwise }\end{cases}$

In model (8), image vibrates along the gradient direction of the zero crossing point which belongs to $G_{\sigma} * I_{\eta \eta}$. When $\left|G_{\sigma} * \nabla I\right|<\tau$, the first term for anisotropic diffusion is predominant; when $\left|G_{\sigma} * \nabla I\right| \geq \tau$, the second term for edge enhancement is the dominant one. $\alpha_{\gamma}$ and $\alpha_{e}$ control the intensity of anisotropic diffusion and shock filter. The bigger $\alpha_{\gamma}$ and $\alpha_{e}$, the stronger the control has.

\section{B. Model of complex shock filter considering the second} derivative amplitude and merged with time dependence

For shock filter formula (1) with 2D signal, if $F(s)=\operatorname{sign}(s)=\operatorname{sign}\left(I_{\eta \eta}\right)$, then both sides of the zero crossing point will have equal weight, which is not conducive to sharpen edges. Due to both the second 
derivative and the first derivative have large amplitude around the zero crossing point, we can consider using the second derivative amplitude to control the shock velocity and choosing $F(s)=2 \arctan (a s) / \pi$. At this time on both sides of the edge the change of gray value of image can be softly controlled and the appearing false piecewise constant area can be eliminated. Then we get improved model proposed by Guy Gilboa [2, 3]:

$$
I_{t}=-\frac{2}{\pi} \arctan \left(a I_{\eta \eta}\right)|\nabla I|+\lambda I_{\eta \eta}+\lambda I_{\xi \xi}
$$

Where, $\lambda=r e^{i \theta}$ is a plural and $\lambda$ is a real number. When compared with distant relative smooth area, the second derivative and the first derivative of edge area near the zero crossing point have large amplitude, which makes it be sharpened faster.

It is hoped to reduce the impact of shock in the process at the very beginning and at the same time noise is mainly smoothed off by diffusion process. With the evolution goes by, the error inflection point caused by noise has been greatly reduced, which can sharpen the edge through enhancing shocked parts. So, a simple method is to multiply the second derivative of shock part by time $t$, then we get:

$$
I_{t}=-\frac{2}{\pi} \arctan \left(a I_{\eta \eta} t\right)|\nabla I|+\lambda I_{\eta \eta}+\lambda I_{\xi \xi}
$$

Combine Eq.(3) with Eq.(10), then we get:

$$
I_{t}=-\frac{2}{\pi} \arctan (a \operatorname{Im}(I) / \theta)|\nabla I|+\lambda I_{\eta \eta}+\lambda I_{\xi \xi}
$$

\section{RESULT AND ANALYSIS OF CREASE DETECTION FOR FEATHER'S QUILL}

\section{A. Comparison on de-noising and enhancement of variety of models}

In order to compare the effect and the advantages of different models for de-noising and enhancement, experiment will take synthetic crease image with size of $60 \times 60$ (as shown in Fig.1), all the model parameters has been optimized by imitating the method of section 5.2 and the iterations are 20 .

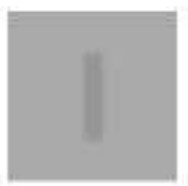

(a)

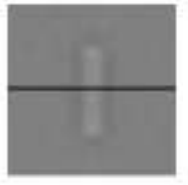

(e)

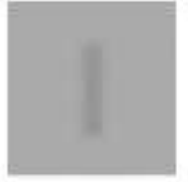

(i)

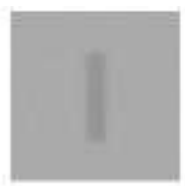

(b)

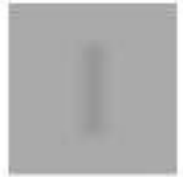

(f)

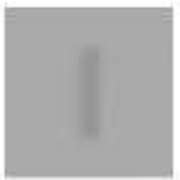

(j)

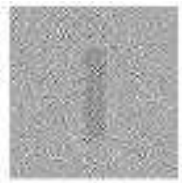

(c)

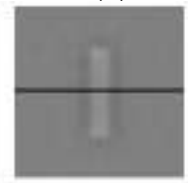

(g)

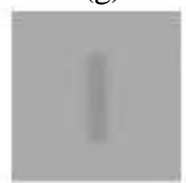

(k)

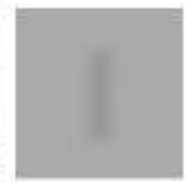

(d)

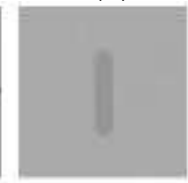

(h)

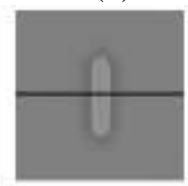

(1)

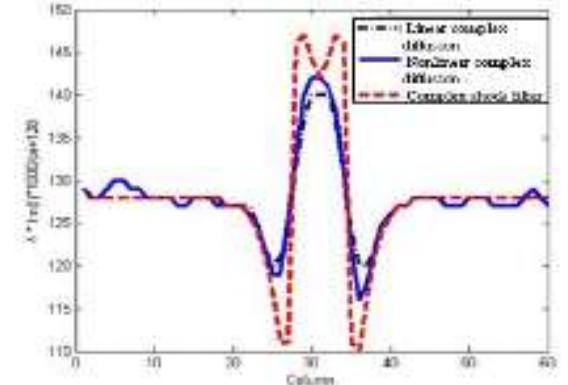

(m)

Figure 1. comparison on de-noising and enhancement of various model

(a) original fuzzy image (b) original adding noise fuzzy image (c) result of formula(1) (d) real part of formula(2)'s result (e) imaginary part of formula(2)'s result (f) real part of formula(5)'s result (g) imaginary part of formula(5)'s result (h) result of ALV formula(6) (i) result of CA formula(7) (j) result of Kornprobst formula(8) (k) real part of formula(11)'s result (1) imaginary part of formula(11)'s result $(\mathrm{m})$ gray distribution of the 30th line in Fig.1 (e)、 (g)、(l)

It is clear that when weak noise exists in image, image enhancement algorithm of shock filter (1) will obviously enlarge noise, the effect of image enhancement is not good (Fig.1 (c)); The real part of Linear complex diffused formula (2) is approximate to $I_{0}$ Gaussian convolution. With time goes by, the real part will de-noise as well as make the edge gradually fuzzy, until the crease disappears at last. (Fig.1 (d)); Real part of nonlinear complex diffusion formula (5) is the smoothed image. With the increase of the number of diffusion, the edge and crease in real part of nonlinear complex diffusion are gradually polished (Fig.1 (f)). ALV formula (6) can remove noise as well as enhance the edge well, but false piecewise constant area appears, namely among different images area there exists discontinuity, which looks unnatural (Fig.1 (h)). However Fig.1 (i) (k), which representing result of CA formula (7), Kornprobst formula (8) and complex shock filter formula (11), can keep the edges while de-noising and achieve good visual quality.

TABLE I. DE-NOISING ESTIMATION RESULT SNR OF DIFFERENT MODEL

\begin{tabular}{|c|c|c|c|}
\hline models & SNR(dB) & models & SNR(dB) \\
\hline $\begin{array}{c}\text { original image } \\
\text { with noise }\end{array}$ & 8.191 & $\begin{array}{c}\text { ALV } \\
\text { formula(6) }\end{array}$ & 9.959 \\
\hline $\begin{array}{c}\text { shock filter } \\
\text { formula(1) }\end{array}$ & -16.151 & $\begin{array}{c}\text { CA } \\
\text { formula(7) }\end{array}$ & 17.592 \\
\hline $\begin{array}{c}\text { linear complex } \\
\text { diffusion } \\
\text { formula(2) }\end{array}$ & 11.028 & $\begin{array}{c}\text { Kornprobst } \\
\text { formula(8) }\end{array}$ & 15.766 \\
\hline $\begin{array}{c}\text { nonlinear } \\
\text { complex } \\
\text { diffusion } \\
\text { formula(5) }\end{array}$ & 11.870 & $\begin{array}{c}\text { complex } \\
\text { diffusion } \\
\text { formula(11) }\end{array}$ & 16.972 \\
\hline
\end{tabular}

Table. 1 is the de-noising evaluation results SNR value of the figure 1, the SNR of complex shock filter is $16.972 \mathrm{~dB}$, which is only next to model CA. But detection requires extracting edge information that is not included in 
model CA. Hence model CA is not suitable for crease detection of feather quill. Respectively take the 30th line pixels gray value in Fig.1 (e)、(g)、(l), the pixels gray value distribution respectively shown in Fig.1 (m) as black lineation, blue solid line and red dashed line. Through comparison, it is found that on the both sides of the edge the change of gray difference of imaginary part of complex shock filter corresponding from red dashed line is the largest, with the narrowest edge width and the least noise on both sides of edge, producing the best effect of edge enhancement and de-noising. Therefore, although Fig.1 (1) smooth off partial angular point information, we chooses complex shock filter formula (11) to detect crease of feather's quill.

\section{B. Model parameters determination}

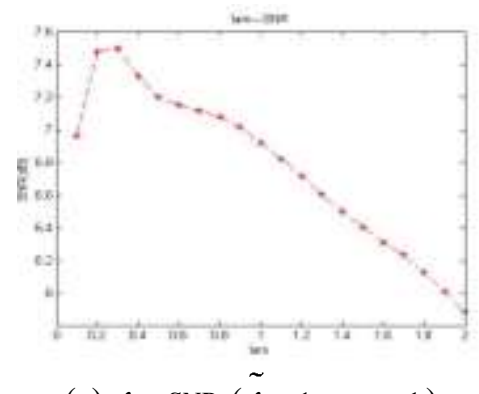

(a) $\lambda-\operatorname{SNR}(\tilde{\lambda}=1, \quad a=1)$

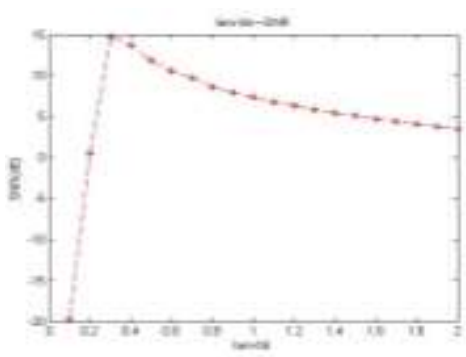

(b) $\tilde{\lambda}-\operatorname{SNR}(\lambda=0.3, \quad a=1)$

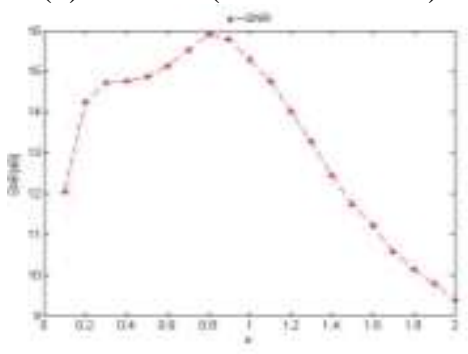

(c) $a-\operatorname{SNR}(\lambda=\tilde{\lambda}=0.3)$

Figure 2. model parameters determination of complex shock filter

Choose complex shock filter (11) to process Fig.1 (a).

Take $\theta=p i / 1000, \lambda=1$ and $a=1$, with 20 iterations. Change $\lambda$, Fig.2 (a) $\lambda-S N R$ curve is gained. It is obviously that when $\lambda=0.3$, value of $S N R$ is biggest. So parameter $\lambda=0.3$ is determined. Next still keep $a=1$ and change $\lambda$. Similarly, when $\lambda=0.3$, value of $S N R$ is biggest, thus parameter $\lambda=0.3$ is determined(as shown in Fig.2(b)). While $\lambda=\lambda=0.3$, value of $a$ is changed, we get curve $a-S N R$ shown in Fig.2(c). It is seen that

when $a=0.8$, value of $S N R$ is the biggest. Hence we take $a=0.8$. Other model parameters can be optimized and determined following this method.

\section{Crease detection of feather's quill}

Through above theoretical analysis, we take the gray image of feather quill with crease as experimental subject. The processing results are shown in Fig.3 and the selected model parameters and iterations are the same with figure 1 .

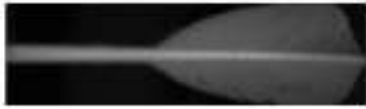

(a)

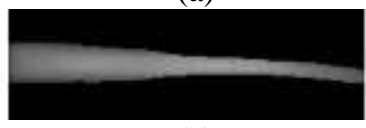

(c)

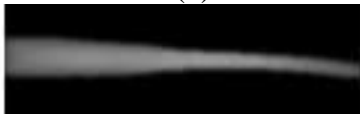

(e)

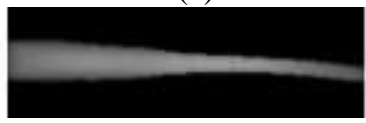

(g)

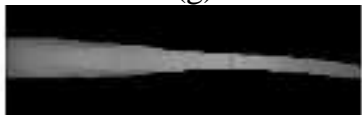

(i)

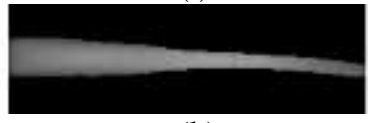

(k)

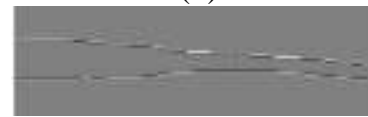

(m)

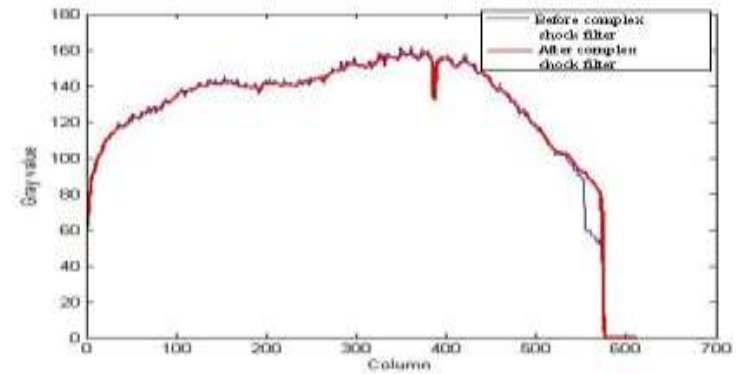

Figure 3. crease detection of feather's quill

(a) original feather image (b) feather quill (c) enlarge feather quill for 3 times in longitudinal (d) result of shock filter formula(1) (e) real part of linear complex diffusion formula(2) (f) imaginary part of linear complex diffusion formula(2) $(\mathrm{g})$ real part of nonlinear complex diffusion formula(5) (h) imaginary part of nonlinear complex diffusion formula(5) (i) result of ALV formula(6) (j) result of CA formula(7) (k) result of Kornprobst formula(8) (1) real part of complex shock filter formula(11) (m) imaginary part of complex shock filter formula(11) (n) gray comparison before and after complex shock filter while go through crease and take a line of pixel in Fig.3 (c)、(1) 
Fig.3 (a) is original image of feather and (b) is the separated rod part of feather [8,9]. With low resolution, the observed feather crease is unobvious in Fig.3 (b); In order to improve image resolution to help detect crease of feather's quill, feather rod can be amplified through linear interpolation in the longitudinal. Fig.3 (c) is the result of feather rod's linear interpolation amplified in the longitudinal with 3 times; Fig. 3 (d) $\sim(\mathrm{m})$ is the result processed by various shock and diffusion model. By comparison it is seen that visual quality of Fig.3 (j) (l) are better, and Fig.3 (1) is the best, for it enhances crease edge as well as de-noises. In Fig.3 (m), detailed edge information reflected in imaginary part is very clearly; Besides, in Fig.3 (c) and (1), take one line of pixels that go through crease, shown as Fig.3 (n), thin blue line and thick red line respectively are the pixel gray distribution before and after complex shock filter. From Fig.3 (n) we can see that the selected complex shock filter formula (11) in this paper can splendidly solve the problem of denoising and edge enhancement for crease image of feather's quill.

Make the clear imaginary part image after complex shock filter (namely Fig.3 (m)) binarization, Fig.4 (a) is got; meanwhile, respectively extract edge image of Fig.3 (c) with noise before complex shock filter by means of Canny、Log、Zerocross, and the results are as shown in Fig.4 (b) (d). From comparison we know that edge extraction from imaginary part image after complex shock filter is the best one.

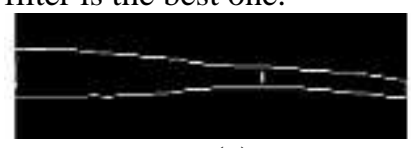

(a)

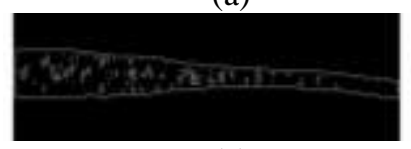

(c)

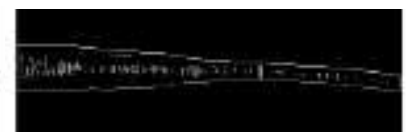

(b)

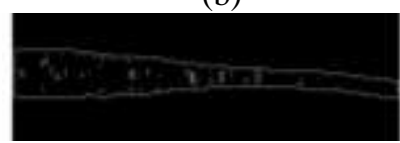

(d)

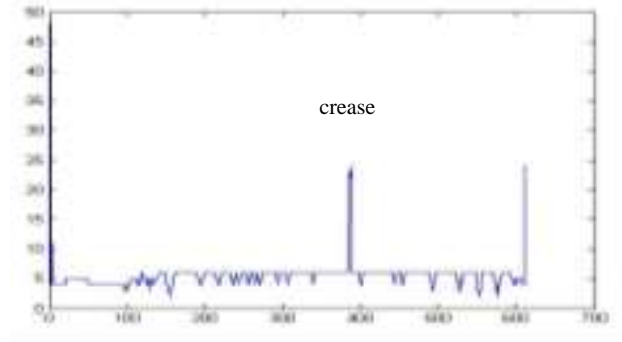

(e)

Figure 4. edge extraction of feather quill and crease detection

(a) Fig.3 (m) binarization (b) edge extration of Fig.3 (c) with Canny (c) edge extration of Fig.3 (c) with Log (d) edge extration of Fig.3 (c) with Zerocross (e) accumulated sum distribution of Fig.4 (a) along normal direction of feather's quill

Finally, according to the characteristic that crease is almost parallel to normal direction of feather's quill, accumulate Fig.4 (a) along normal direction of feather's quill, then distribution curve of accumulated sum (namely Fig.4 (e)) is got. It is clear that if neglecting the effect of two ends of feather quill, crease is corresponding to the maximum value position of Fig.4 (e). Whether the maximum value surpasses a certain threshold (according to a lot of crease test statistics, this threshold value can be 12) can decide whether the crease of feather quill exist or not. This judgment method is simple and of high reliability.

\section{THE CONCLUSION}

This paper analyses both principle of de-noising and sharpen enhancement and existing problem, offering a method of testing feather quill's crease by coupling shock filter and complex diffusion. The process of coupling uses image second derivative to control shock speed, combined with time dependence, then develop into the shock filter controlled by the imaginary part of complex diffusion, and the shock filter process needs no convolution operation. Simulation results show that the method used in this thesis achieves good effect of de-noising in the real part image of feather's quill after processed by complex shock filter, and edge in the real part image is effectively enhanced instead of blurred, with sound visual effect; The imaginary part enhances the edge greatly, which is much better than operates of Canny, Log, Zerocross etc. Make the imaginary part image after complex shock filter binarization and accumulated along normal direction of feather's quill. Existence of crease can be judged by accumulation sum value. Through a lot of experiments on crease of feather's quill, the method discussed in this thesis can be effectively used for crease detection of feather's quill.

\section{REFERENCES}

[1] S.J. Osher, L.I. Rudin. Feature-Oriented Image enhancement using Shock Filters[J]. SIAM J. Numer. Anal. 1990(27):919-940.

[2] Guy Gilboa, Nir Sochen, Zeevi Y Y. Image enhancement and denoising by complex diffusion processes[J]. IEEE Trans. On Pattern Analysis and Machine Intelligence, 2003, 28(8):1020-1036.

[3] Guy Gilboa, Nir A. Sochen, Zeevi Y Y. Regularized shock filters and complex diffusion[C]. In: Proceedings of the 7th European Conference on Computer Vision, LNCS 2350, Springer-Verlag, 2002:399-413.

[4] Perona P, Malik J. Scale space and edge detection using anisotropic diffusion[J]. IEEE Transactions on Pattern Analysis and Machine Intelligence, 1990, 12:629-639.

[5] ALVAREZ L, MAZORRA L. Signal and image restoration using shock filters and anisotropic diffusion[J]. SIAM J. Numer. Anal. 1994, 31(2):590-605.

[6] COULON O, ARRIDGE S R. Dual echo MR image processing using multi-spectral probabilistic diffusion coupled with shock filters[C]. MIUA 2000, British Conference on Medical Image Understanding and Analysis, London, United-Kingdom, 2000:256264.

[7] KORNPROBST P, DERICHE R, AUBERT G. Image coupling, restoration and enhancement via PDE's[C]. Proc. Int. Conf. on Image Processing 1997: Santa-Barbara (USA), 1997:458-461.

[8] Liu Hongjiang, Wang Renhuang, He Zuihong,etc. Slender Object Extraction for Image Segmentation Based on Centerline Snake Model[J]. Photoelectric engineering, 2011, 55 (12,):124-129.

[9] Liu Hongjiang, Wang Renhuang, Li Xuecong. Improved algorithm of feather image segmentation based on active contour model[J]. Computer application, 2011, 31 (8):2246-2248.

[10] M. Nitzberg, T. Shiota. Nonlinear image filtering with edge and corner enhancement $[\mathrm{J}]$. IEEE Transactions on Pattern Analysis and Machine Intelligence, 1992, 14(8):826-833. 